(2)

\title{
Efficacy of the small molecule inhibitor of Lipid II BASO0 127538 against Acinetobacter baumannii
}

This article was published in the following Dove Press journal:

Drug Design, Development and Therapy

8 August 2014

Number of times this article has been viewed

\section{Erik PH de Leeuw \\ Institute of Human Virology, \\ University of Maryland School \\ of Medicine, Baltimore, MD, USA}

Correspondence: Erik PH de Leeuw Institute of Human Virology of the University of Maryland Baltimore School of Medicine, 725 West Lombard Street, Baltimore, MD 21201, USA

Tel +l 4I0 7061970

Fax + I 4107067583

Email edeleeuw@som.umaryland.edu
Objective: To test the activity of a small molecule compound that targets Lipid II against Acinetobacter baumannii.

Methods: Susceptibility to small molecule Lipid II inhibitor BAS00127538 was assessed using carbapenem- and colistin-resistant clinical isolates of $A$. baumannii. In addition, synergy between colisitin and this compound was assessed.

Results: Small molecule Lipid II inhibitor BAS00127538 potently acts against A. baumannii and acts synergistically with colistin.

Conclusion: For the first time, a compound that targets Lipid II is described that acts against multi-drug resistant isolates of $A$. baumannii. The synergy with colistin warrants further lead development of BAS00127538.

Keywords: Lipid II, Acinetobacter baumannii, drug development

\section{Introduction}

Lipid II is an essential precursor in cell wall biosynthesis, and is a validated antibacterial drug target. It is comprised of a hydrophilic head group that includes a peptidoglycan subunit composed of $\mathrm{N}$-acetylglucosamine and $\mathrm{N}$-acetylmuramic acid. The cell wall of all bacteria comprises this polymer of alternating amino sugars. The peptidoglycan layer of Gram-negative bacteria is generally much smaller than the Gram-positive cell wall (1.5 versus $\sim 20$ layers). ${ }^{1}$

The natural glycopeptide antibiotic vancomycin was the first compound discovered to kill bacteria by targeting Lipid II. Currently, vancomycin serves as a principal treatment for infections caused by all major Gram-positive pathogens, including methicillinresistant Staphylococcus aureus. However, resistance to vancomycin is increasing and a number of vancomycin insensitive strains have been described. Two resistance mechanisms have been described in these strains: affinity trapping of vancomycin by increased production of cell wall monomers, and change in metabolism and structure of teichoic acid. ${ }^{2}$ Reported resistance in clinical isolates resulted from replacing the carboxy terminal D-alanyl-D-alanine target for vancomycin by D-alanyl-D-lactate. ${ }^{3}$ Resistance to vancomycin appeared over 20 years, predominantly because vancomycin was reserved as a last resort antibiotic to prolong development of resistance. The vancomycin derivative telavancin (Vibativ ${ }^{\circledR}$; Theravance Biopharma US, Inc., South San Francisco, CA, USA) was approved by the Food and Drug Administration for complicated skin and skin structure infections in 2009 and more recently for hospitalacquired pneumonia when alternative treatments are not suitable. Two next-generation 
vancomycin-derived glycopeptides that bind Lipid II are currently in clinical trials: dalbavancin hydrochloride (Durata Therapeutics, Inc., Chicago, IL, USA) and oritavancin (The Medicines Company, Parsippany-Troy Hills, NJ, USA).

Resistance against commonly used classical antibiotics has emerged in all major classes of both Gram-positive and Gram-negative pathogens, including S. aureus, Enterococcus faecium, Pseudomonas aeruginosa, and Acinetobacter baumannii. ${ }^{4,5}$ Multi-drug resistant $A$. baumannii is an increasingly important Gram-negative pathogen, particularly in hospital settings. ${ }^{4}$ The types of infections caused by this pathogen include pneumonia, endocarditis, bacteremia, skin and soft tissue infections, and meningitis., ${ }^{4,6-8}$ Factors that contribute to pathogenesis of this organism include iron acquisition, membrane polysaccharides and vesicles, biofilm formation, and penicillin-binding proteins. ${ }^{9}$ We have recently described small molecule inhibitors of Lipid II for the first time. ${ }^{10}$ Our most promising compound, BAS00127538, was highly active against Gram-positive species and showed activity against Gram-negative species also, albeit reduced. This compound binds to Lipid II differently than other compounds, affects cell wall biosynthesis, and showed efficacy in vivo. ${ }^{10}$ Since vancomycin, dalbavancin, and oritavancin fail to demonstrate any notable activity against $A$. baumannii, we tested activity of our lead small molecule Lipid II inhibitor BAS00127538 (Figure 1) against this species.

\section{Materials and methods}

\section{Strains}

Twelve isolates of carbapenem- and/or colistin-sensitive or resistant $A$. baumannii were obtained from the Laboratory of Pathology, University of Maryland Baltimore School of Medicine (Baltimore, MD, USA). All isolates were

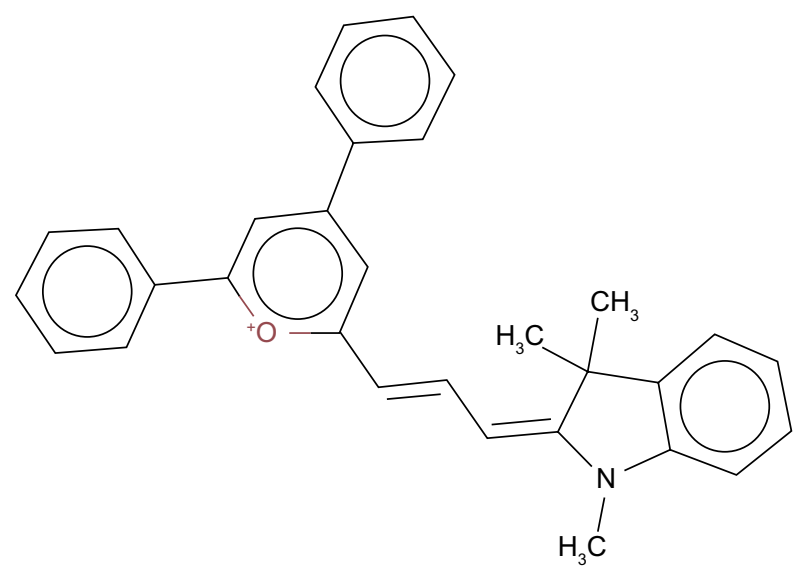

Figure I Chemical structure of the di-phenyl-tri-methyl-indolene-pyranilium compound BASOOI 27538. characterized by Etest (meropenem) or minimum inhibitory concentration (MIC) (colistin) according to the Clinical and Laboratory Standards Institute guidelines as indicated in Table 1. A. baumannii ATCC 19606 was included as a reference strain for quality control testing.

\section{Antimicrobial agents}

Colistin and meropenem were obtained from LKT Laboratories, Inc., (St Paul, MN, USA). Vancomycin was purchased from Sigma-Aldrich Co (St Louis, MO, USA). BAS00127538 was obtained from Asinex (Winston-Salem, $\mathrm{NC}, \mathrm{USA})$.

\section{Synergy screen}

Synergy was determined by checkerboard method. ${ }^{11}$ The stock solutions and serial two-fold dilutions of each drug to at least double the MIC were prepared according to the recommendations of the National Committee for Clinical Laboratory Standards immediately prior to testing. A total of $50 \mu \mathrm{L}$ of Mueller-Hinton broth was distributed into each well of the microdilution plates. The first antibiotic of the combination was serially diluted along the ordinate, while the second drug was diluted along the abscissa. An inoculum equal to a $0.5 \mathrm{McFarland}$ turbidity standard was prepared from each $A$. baumannii isolate in Mueller-Hinton broth. Each microtiter well was inoculated with $100 \mu \mathrm{L}$ of a bacterial inoculum of $5 \times 10^{5} \mathrm{CFU} / \mathrm{mL}$, and the plates were incubated at $37^{\circ} \mathrm{C}$ for 24 hours under aerobic conditions. According to the National Committee for Clinical Laboratory Standards guidelines for broth microdilution, the MIC was defined as the lowest concentration of antibiotic that completely inhibited the growth of the organism as detected with the naked eye. The $\Sigma$ FICs (fractional inhibitory concentrations) were calculated as follows: $\Sigma$ FIC $=$ FIC A + FIC B, where FIC A is the MIC of drug $\mathrm{A}$ in the combination/MIC of drug A alone, and FIC B is the $\mathrm{MIC}$ of drug B in the combination/MIC of drug B alone.

\section{Results}

Table 1 shows a summary of the MIC for BAS00127538 in comparison to colisitin, meropenem, and vancomycin, as well as synergy studies for BAS00127538 with colistin. Compound BAS00127538 showed activity against all isolates tested (MIC 2-8 $\mu \mathrm{g} / \mathrm{mL}$ ), regardless of their resistance profile. As expected, vancomycin did not show activity against these isolates. Table 1 also shows a summary of the colistin and BAS00127538 synergy testing. By the screening method, BAS00127538 synergy with colistin was found for $11 / 13(84.6 \%)$ strains with no apparent correlation with 
Table I Broth microdilution susceptibility testing and synergy for BAS00I 27538 and comparators

\begin{tabular}{|c|c|c|c|c|c|}
\hline Strain & Colisitin $^{a}$ & Carbapenem $^{\mathrm{b}}$ & $\begin{array}{l}\text { Vancomycin } \\
\text { (MIC, } \mu \mathrm{g} / \mathrm{mL} \text { ) }\end{array}$ & $\begin{array}{l}\text { BAS00I } 27538 \\
(M I C, \mu g / m L)^{c}\end{array}$ & $\begin{array}{l}\text { BAS00 I } 27538 / \text { colistin } \\
\text { synergy }(\Sigma F I C)^{c, d}\end{array}$ \\
\hline I_2_I & Sensitive & Sensitive & $>128$ & 8 & $<0.5$ \\
\hline I_7_I & Sensitive & Resistant & $>128$ & 4 & $0.5-2.0$ \\
\hline I_8_I & Sensitive & Resistant & $>128$ & 8 & $0.5-2.0$ \\
\hline I_9_I & Sensitive & Resistant & $>128$ & 4 & $<0.5$ \\
\hline I_4_2 & Sensitive & Sensitive & $>128$ & 4 & $<0.5$ \\
\hline I_5_2 & Sensitive & Sensitive & $>128$ & 2 & $<0.5$ \\
\hline 1_9_2 & Resistant & Resistant & $>128$ & 4 & $<0.5$ \\
\hline I_10_2 & Sensitive & Sensitive & $>128$ & 2 & $<0.5$ \\
\hline I_II_2 & Sensitive & Sensitive & $>128$ & 8 & $<0.5$ \\
\hline 2_9_1 & Resistant & Resistant & $>128$ & 4 & $<0.5$ \\
\hline $2 \_2 \_2$ & Resistant & Resistant & $>128$ & 2 & $<0.5$ \\
\hline 8_5_1 & Resistant & Resistant & $>128$ & 4 & $<0.5$ \\
\hline ATCC 19606 & Sensitive & Sensitive & $>128$ & 2 & $<0.5$ \\
\hline
\end{tabular}

Notes: Experiments were carried out according to CLSI standards. ${ }^{24}$ a MIC $\leq 2$ Sensitive; MIC $\geq 4$ Resistant; ${ }^{b}$ meropenem zone size $\leq 14$ mm Sensitive; zone size $\geq 18$ mm Resistant; 'MIC was determined according to CLSI guidelines; ${ }^{\mathrm{d} v a l u e s}$ represent five individual experiments.

Abbreviations: CLSI, Clinical and Laboratory Standards Institute; FIC, fractional inhibitory concentration; MIC, minimum inhibitory concentration.

colistin or carbapenem resistance profiles. Synergy determined by this method resulted in a four- to eight-fold reduction in the MIC of BAS00127538 and a two- to four-fold reduction in MIC for colistin with the exception of the 1_7_1 and 1_8_1 isolates, against which synergy was not observed.

\section{Discussion}

With the increases of drug resistance, clinical use of polymyxins, such as colistin, has seen a revival. ${ }^{12,13}$ Here, we report for the first time any Lipid II inhibitor with activity against A. baumannii. Compound BAS00127538 specifically and uniquely interacts with the $\mathrm{N}$-acetyl muramic acid moeity and isoprenyl tail of Lipid II with additional predicted interactions with the Lipid II phosphate moiety. ${ }^{10}$ BAS00127538 was found to affect cell wall biosynthesis with membrane perturbation as a likely secondary mechanismof-action. ${ }^{10}$ Colistin is believed to act by competitive displacement of divalent cations from the phosphate groups of membrane lipids and by binding to lipopolysaccharides in the outer cell membrane of Gram-negative bacteria..$^{14,15}$ Binding leads to disruption of the outer cell membrane in a detergent-like fashion, resulting in leakage of intracellular contents and bacterial death. Colistin binds to the Lipid A component of lipopolysaccharides and alterations to the Lipid A, or complete loss of all lipopolysaccharides, are associated with colistin resistance. ${ }^{16,17}$ The addition of phosphoethanolamine to Lipid A as a resistance mechanism has been mapped to mutations in the PmrAB two-component system. ${ }^{18}$ Other modifications, such as reduction of net charge of Lipid A with 4-amino-4-deoxy-L-arabinose, have been described for other species, including Escherichia coli, Klebsiella pneumoniae, and P. aeruginosa. ${ }^{19} \mathrm{BAS} 00127538$ shows activity against E. coli (MIC $4 \mu \mathrm{g} / \mathrm{mL}$ ), and P. aeruginosa (MIC $8 \mu \mathrm{g} / \mathrm{mL}$ ). Alternative modes of action, other than acting on bacterial cell membrane, have been reported for polymyxins also. ${ }^{20}$

Activity of BAS00127538 against colistin-resistant isolates as well as the observed synergy between colistin and BAS00127538 indicates that both compounds differ in mechanism-of-action, despite the apparent structural resemblance between their respective targets, Lipid A and Lipid II. Hydrophilic antibiotics such as rifampicin, carbapenems, and glycopeptides, including the Lipid II-binding natural compound vancomycin, can work synergistically with colistin. ${ }^{21}$ However, no other compound that targets Lipid II is active against $A$. baumannii. For example, vancomycin and its derivative telavancin reportedly lack potency against A. baumannii. ${ }^{22,23}$ This lack of potency is attributed to inaccessibility of these compounds to the peptidoglycan layer. Inaccessibility is due to impermeability of the Gram-negative outer to these drugs. ${ }^{22}$ Synergism between colistin and these Lipid II-binding drugs has been described..$^{22,23}$ In these studies, colistin potentiates the efficacy of these compounds against $A$. baumannii by permeabilizing the Gram-negative membrane. Small molecule Lipid II inhibitors such as BAS00127538 are more likely to reach their target directly by themselves and are further potentiated by colistin in a similar manner as vancomycin or its derivatives. Our findings suggest that small molecule inhibitors of Lipid II can be developed into a novel class of broad-spectrum therapeutics against clinically relevant Gram-positive and Gram-negative pathogens. 


\section{Conclusion}

The relentless rise in antibiotic resistance combined with underinvestment in discovery and development of antibacterial agents will severely effect our ability to treat infections in hospitals and the community. There is an urgent need to develop novel, broad-spectrum antibacterial therapeutics to treat infections caused by antibacterial-resistant pathogens. Development of synthetic Lipid II inhibitors such as BAS00127538 as a novel class of antibiotics with a unique mechanism-of-action represents a important approach to meet the critical clinical need for orally active, novel broadspectrum antibacterial agents.

\section{Acknowledgments}

The Laboratory of Pathology, University of Maryland Baltimore School of Medicine is gratefully acknowledged for providing the A. baumannii clinical isolates. This work is supported by the National Institutes of Health grant to the author (Grant R21AI092033).

\section{Disclosure}

The author reports no conflicts of interest in this work. US patent applications \# 61/656,039 and US Non-Provisional Patent Application \# 13/883,564 have been filed (Alexander D MacKerell and Erik PH de Leeuw, Inventors).

\section{References}

1. Breukink E, de Kruijff B. Lipid II as a target for antibiotics. Nat Rev Drug Discov. 2006;5:321-332.

2. Depardieu F, Podglajen I, Leclercq R, Collatz E, Courvalin P. Modes and modulations of antibiotic resistance gene expression. Clinl Microbiol Rev. 2007;20(1):79-114.

3. Weigel LM, Clewell DB, Gill SR, et al. Genetic analysis of a high-level vancomycin-resistant isolate of Staphylococcus aureus. Science. 2003;302(5650):1569-1571.

4. Dijkshoorn L, Nemec A, Seifert H. An increasing threat in hospitals: multidrug-resistant Acinetobacter baumannii. Nat Rev Microbiol. 2007;5(12):939-951.

5. Swartz MN. Hospital-acquired infections: diseases with increasingly limited therapies. Proc Natl Acad Sci U S A. 1994;91(7):2420-2427.

6. Wisplinghoff H, Perbix W, Seifert H. Risk factors for nosocomial bloodstream infections due to Acinetobacter baumannii: a case-control study of adult burn patients. Clin Infect Dis. 1999;28(1):59-66.
7. Murray CK, Roop SA, Hospenthal DR, et al. Bacteriology of war wounds at the time of injury. Mil Med. 2006;171(9):826-829.

8. Krol V, Hamid NS, Cunha BA. Neurosurgically related nosocomial Acinetobacter baumannii meningitis: report of two cases and literature review. $J$ Hosp Infect. 2009;71(2):176-180.

9. McConnell MJ, Actis L, Pachón J. Acinetobacter baumannii: human infections, factors contributing to pathogenesis and animal models. FEMS Microbiol Rev. 2013;37(2):130-155.

10. Varney KM, Bonvin AM, Pazgier M, et al. Turning defense into offense: defensin mimetics as novel antibiotics targeting lipid II. PLoS Pathog. 2013;9(11):e1003732.

11. Orhan G, Bayram A, Zer Y, Balci I. Synergy tests by E test and checkerboard methods of antimicrobial combinations against Brucella melitensis. J Clin Microbiol. 2005;43(1):140-143.

12. Falagas ME, Kasiakou SK. Colistin: the revival of polymyxins for the management of multidrug-resistant gram-negative bacterial infections. Clin Infect Dis. 2005;40(9):1333-1341.

13. Li J, Nation RL, Turnidge JD, et al. Colistin: the re-emerging antibiotic for multidrug-resistant Gram-negative bacterial infections. Lancet Infect Dis. 2006;6(9):589-601.

14. Li J, Nation RL, Milne RW, Turnidge JD, Coulthard K. Evaluation of colistin as an agent against multi-resistant Gram-negative bacteria. Int $J$ Antimicrob Agents. 2005;25(1):11-25.

15. Landman D, Georgescu C, Martin DA, Quale J. Polymyxins revisited. Clin Microbiol Rev. 2008;21(3):449-465.

16. Pelletier MR, Casella LG, Jones JW, et al. Unique structural modifications are present in the lipopolysaccharide from colistinresistant strains of Acinetobacter baumannii. Antimicrob Agents Chemother. 2013;57(10):4831-4840.

17. Moffatt JH, Harper M, Harrison P, et al. Colistin resistance in Acinetobacter baumannii is mediated by complete loss of lipopolysaccharide production. Antimicrob Agents Chemother. 2010;54(12):4971-4977.

18. Beceiro A, Llobet E, Aranda J, et al. Phosphoethanolamine modification of lipid A in colistin-resistant variants of Acinetobacter baumannii mediated by the pmrAB two-component regulatory system. Antimicrob Agents Chemother. 2011;55(7):3370-3379.

19. Raetz CR, Whitfield C. Lipopolysaccharide endotoxins. Annu Rev Biochem. 2002;71:635-700.

20. Brogden KA. Antimicrobial peptides: pore formers or metabolic inhibitors in bacteria? Nat Rev Microbiol. 2005;3(3):238-250.

21. Petrosillo N, Ioannidou E, Falagas ME. Colistin monotherapy vs combination therapy: evidence from microbiological, animal and clinical studies. Clin Microbiol Infect. 2008;14(9):816-827.

22. Gordon NC, Png K, Wareham DW. Potent synergy and sustained bactericidal activity of a vancomycin-colistin combination versus multidrug-resistant strains of Acinetobacter baumannii. Antimicrob Agents Chemother. 2010;54(12):5316-5322.

23. Hornsey M, Longshaw C, Phee L, Wareham DW. In vitro activity of telavancin in combination with colistin versus Gram-negative bacterial pathogens. Antimicrob Agents Chemother. 2012;56(6):3080-3085.

24. Clinical and Laboratory Standards Institute. Methods for Dilution Antimicrobial Susceptibility Tests for Bacteria That Grow Aerobically; Approved Standard - Eighth Edition. 2009.
Drug Design, Development and Therapy

\section{Publish your work in this journal}

Drug Design, Development and Therapy is an international, peerreviewed open-access journal that spans the spectrum of drug design and development through to clinical applications. Clinical outcomes, patient safety, and programs for the development and effective, safe, and sustained use of medicines are a feature of the journal, which

\section{Dovepress}

has also been accepted for indexing on PubMed Central. The manuscript management system is completely online and includes a very quick and fair peer-review system, which is all easy to use. Visit http://www.dovepress.com/testimonials.php to read real quotes from published authors. 